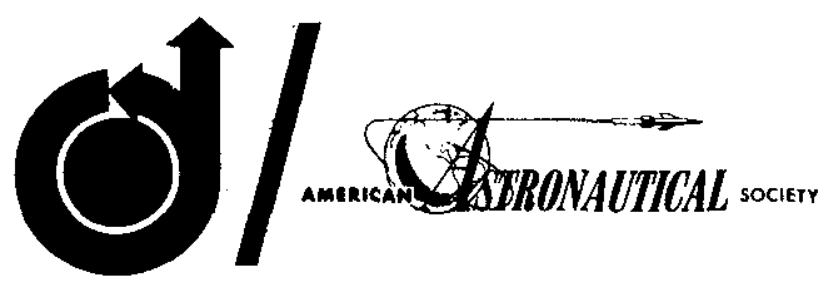

\title{
OPTIMAL TRAJECTORY COORDINATE-MULTIPLIER SYSTEMS WITH CONSTANT OF THE MOTION COMPONENTS
}

by

WILLIAM E. MINER

NASA Electronics Research Center

Cambridge, Massachusetts

and

WILLIAM F. POWERS

The University of Michigan

Ann Arbor, Michigan

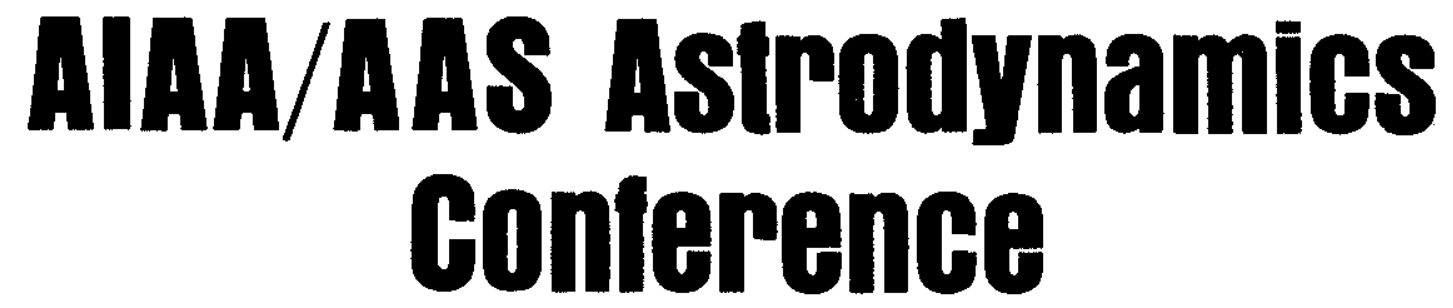

PRINCETON, NEW JERSEY/AUGUST 20-22, 1969 


\author{
William E. Miner* \\ NASA-Electronics Research Center \\ Cambridge, Massachusetts \\ William F. Powers*** \\ The University of Michigan \\ Ann Arbor, Michigan
}

\begin{abstract}
A relatively well-known property of continuously thrusting optimal trajectories is that there exists a vector constant of the motion which is completely analogous to the angular momentum integral of the three-body problem. In this analysis the range of applicability of the integral is extended in two ways. First, it is shown that there exists a large class of coordinate systems such that a conjugate Lagrange multiplier is a constant of the motion, and a method for generating systems with this property is presented. Such a method is applicable to the problem of defining nonstandard state variables for which a conjugate multiplier is a constant of the motion. Second, a nontrivial canonical transformation is used to generate a new system of canonical variables such that three of the variables are strictly functions of the components of the vector integral. Thus, the three variables are constants of the motion for the optimal trajectory problem. In addition, the canonical transformation is effected in such a way that all the new canonical variables are constants of the motion for the coast-arc problem.
\end{abstract}

\section{Introduction}

An attractive property of continuously thrusting optimal trajectory problems is that there exists a vector constant of the motion( $(1)$ identical in form to the angular momentum integral of the three-body problem. In the planar problem the integral reduces to a scalar which is actually the Lagrange multiplier conjugate to the range angle in a polar coordinate formulation. One can generalize this fact to show that there exists a constant Lagrange multiplier in nonplanar problems if the coordinate system is cylindrical or spherical.

The purpose of this paper is to extend the range of applicability of the vector integral in the following ways: (1) it will be shown that there exists a large class of nonplanar coordinate systems such that a conjugate Lagrange multiplier is a constant of the motion and a method for developing

TThis research was supported in part by NASA under Contract No. NASr 54(06) and Grant No. NGR 23-005-329.

*Chief, Computational Theory and Techniques Branch.

$* *$

Assistant Professor, Department of Aerospace Engineering. such systems will be presented; and (2) a canonical transformation will be effected in such a way that the new canonical system possesses three components which are strictly functions of the three components of the vector integral, i.e., three of the new variables are constants of the motion.

\section{Basic Theory}

In this section the basic elements of canonical transformation theory necessary for the developments in the following sections are reviewed. It is assumed that the optimal trajectory problem under consideration is nonsingular and that the resultant Hamiltonian formulation of the problem has been transformed into a new Hamiltonian system which is isomorphic to the systems of classical mechanics $(2)$, i. e., $H=\sum_{i=1}^{n} \lambda_{i} f_{i}(t, x, \lambda)$ does not contain the control variables.

DEFINITION II. 1: Let $\{\mathrm{X}(\mathrm{x}, \lambda, t), \Lambda(\mathrm{x}, \lambda, \mathrm{t})\} \in \mathrm{C}^{2}$ be a nonsingular transformation. If for "every" Hamiltonian $\mathrm{H}(\mathrm{x}, \lambda, t)$ there exists a Hamiltonian $\mathrm{K}(\mathrm{X}, \Lambda, t)$, then the transformation is said to be canonical.

Note that the word "every" is emphasized in the above definition. The definition does not say that each transformation which preserves Hamiltonian form is canonical, but only those which preserve Hamiltonian form and are independent of the Hamiltonian function. Also, Definition II. 1 is not a good "working" definition, i.e., one cannot check every Hamiltonian function. However, this definition leads to the following workable conditions for checking and generating canonical transformations.

PROPERTY II.1: (i) (Poisson brackets) The transformation $\{X(x, \lambda, t), \Lambda(x, \lambda, t)\}$ is canonical if and only if there exists a nonzero scalar constant $\mu$ such that $\left\{X_{i}, X_{j}\right\}=0,\left\{\Lambda_{i}, \Lambda_{j}\right\}=0$, and $\left\{\Lambda_{i}, X j\right\}=\mu \delta_{i j}$ for each $i, j=l, \ldots, n$, where $\{A, B\}$ $\equiv \sum_{k=1}^{n}\left(\frac{\partial A}{\partial \lambda_{k}} \frac{\partial B}{\partial x_{k}}-\frac{\partial A}{\partial x_{k}} \frac{\partial B}{\partial \lambda_{k}}\right)$. (ii) (Generating functions) If there exists a scalar function $\mathrm{S}$ such that

$$
\sum_{i=1}^{n} \lambda_{i} \dot{x}_{i}-H(x, \lambda, t)=\sum_{i=1}^{n} \Lambda_{i} \dot{X}_{i}-K(X, \Lambda, t)+\frac{d S}{d t} \text {. }
$$

then the transformation $\{x(x, \lambda, t), \Lambda(x, \lambda, t)\}$ is canonical.

With time as the independent variable, Eq. (I) can be expressed equivalently as

$$
\delta S=\sum_{i=1}^{n}\left(\lambda_{i} \delta x_{i}-\Lambda_{i} \delta x_{i}\right)
$$




$$
K=\frac{\partial S}{\partial t}+H .
$$

If the new Hamiltonian, $K$, is identically zero, then $\mathrm{Eq}$. (3) becomes the Hamilton-Jacobi equation and the new variables $\mathrm{X}, \Lambda$ are constants of the motion.

Equations (2) and (3) are useful for defining the following class of canonical transformations.

DEFINITION II. 2: A canonical transformation in which $\frac{\partial S}{\partial t}=0$ and $\delta S=0$ is called a homogeneous canonical transformation. If, in addition, $\mathrm{n}$ independent relations between $\left\{x_{1}, \ldots, x_{n}\right\}$ and $\left\{x_{1}\right.$, $\left.\ldots, x_{n}\right\}$ are specified, then the transformation is called an extended point-transformation.

The importance of extended point-transformations in the analysis of optimal control problems is demonstrated by the following property.

PROPERTY II. 2: Let $\mathbf{x}=\phi(X)$ be a nonsingular transformation between the coordinates of two Hamiltonian systems defined by $H=\sum_{i=1}^{n} \lambda_{i} f_{i}(t, x, \lambda)$ and $K=\sum_{i=1} \Lambda_{i} F_{i}(t, X, \Lambda)$. Then; the time independent. Lagrange multiplier transformation between the two systems is defined by the $n$-equations

$$
\Lambda_{i}=\sum_{j=1}^{n} \lambda_{j} \frac{\partial \phi_{j}}{\partial X_{i}} . \quad(i=1, \ldots, n)
$$

Property II. 2 has a number of important consequences. First of all, it tells us how to determine very simply the Lagrange multiplier transformation between any two coordinate formulations of the same optimal trajectory problem. Secondly, it tells us that these transformations are linear with respect to the Lagrange multipliers. This fact is important since the components of the vector integral for the optimal trajectory problem are linear in the multipliers. In Reference 3, Whittaker presents a method for performing a canonical transformation which transforms a scalar integral linear in the multipliers into a new multiplier variable. Thus, this method has an immediate application in trajectory analysis.

\section{Coordinate Systems With One Constant Multiplier}

In the previous section it was noted that the relationship between the Lagrange multipliers for two sets of state variables is linear. This fact motivates the following question: "Does there exist a set of state variables such that three of the conjugate multipliers are equal to three independent linear functions of the known constants of the motion?" This question is answered in the following theorem.

THEOREM III. $l$ : Let $A_{1} \bar{i}+A_{2} \bar{j}+A_{3} \bar{k}$ be the known vector integral of the optimal trajectory problem expressed in cartesian coordinates. There does not exist a canonical transformation such that two (or three) of the new canonical variables are independent linear combinations of the $\mathrm{A}_{i}{ }^{\prime} \mathrm{s}$.
This theorem is easily proved by applying the Poisson bracket conditions to all possible linear combinations of the $\mathrm{A}_{\mathrm{i}}{ }^{\prime} \mathrm{s}$. Also, the theorem tells us that if a canonical system of variables with three components dependent only upon the $A_{i}$ 's exists, then at least two of the three components must be nonlinear functions of the $A_{i}{ }^{\prime} s$. This means that such a system will be a hybrid system in the sense that no $n$ of the $2 n$ new variables are natural state variables. The development of such a hybrid system will be discussed in Section IV.

Even though no two linear combinations of the $A_{i}{ }^{\prime} s$ can be transformed into new canonical variables, the method of Whittaker gives us the means for generating a large class of sets of state variables such that a conjugate multiplier in each set is a constant of the motion. The method for generating the new canonical systems is described below.

Consider a Hamiltonian system which possesses an integral linear and homogeneous in the Lagrange multipliers, say

$$
g_{1}(x) \lambda_{1}+\cdots+g_{n}(x) \lambda_{n}=\text { constant. }
$$

Without loss of generality, let $\mathrm{Eq}$. (5) be $\Lambda_{\mathrm{n}}$ in the new $\{\mathrm{X}, \Lambda\}$-system which is to be defined by an extended point-transformation $x=\phi(X)$. Then, by Eq. (4) :

$$
\Lambda_{i}=\sum_{j=1}^{n} \lambda_{j} \frac{\partial \phi_{j}}{\partial X_{i}} .(i=1, \ldots, n)
$$

In order that Eqs. (5) and (6) be consistent, there must exist $n$ independent functions $\phi_{1}(X), \ldots, \phi_{n}(X)$ such that

$$
g_{j}(x)=\frac{\partial \phi_{j}}{\partial X_{n}} . \quad(j=1, \ldots, n)
$$

The existence of these functions is guaranteed by first noting that

$$
d x_{i}=\sum_{j=1}^{n} \frac{\partial \phi_{i}}{\partial x_{j}} d x_{j} \equiv \sum_{j=1}^{n} \phi_{i j} d x_{j},
$$

and then applying the classic integrability theorem for a system of total differential equations! 4 )

To determ ine the functions $\phi_{1}, \ldots, \phi_{n}$, we first observe that

$$
d x_{i}=g_{i}(x) d x_{n}(i=1, \ldots, n)
$$

which implies

$$
\frac{\mathrm{dx}_{1}}{\mathrm{~g}_{1}}=\frac{\mathrm{dx}_{2}}{\mathrm{~g}_{2}}=\ldots=\frac{\mathrm{dx_{n }}}{\mathrm{g}_{\mathrm{n}}}=\mathrm{dx} \mathrm{n}_{\mathrm{n}} .
$$

Note that the only restriction on the functions $\phi_{i}(x)$ is that $\frac{\partial \phi_{i}}{\partial \mathrm{X}_{n}}=\mathrm{g}_{\mathrm{i}}(\mathrm{i}=1, \ldots, \mathrm{n})$. Thus, there exist many point-transformations which satisfy this criterion, and such a transformation can be defined by the following procedure:

(i) Determine $\mathrm{n}-1$ integrals of the system (10), 
and denote these integrals by $X_{1}, \ldots, X_{n-1}$. Thus,

$\mathrm{X}_{\mathrm{i}} \equiv \psi_{\mathrm{i}}\left(\mathrm{x}_{1}, \ldots, \mathrm{x}_{\mathrm{n}}\right)=$ constant. $(\mathrm{i}=1, \ldots, \mathrm{n}-1)$

(ii) Use Egs. (11) to express $n-1$ elements of the set $\left\{x_{1}, \ldots, x_{n}\right\}$ as functions of the $X_{i}{ }^{\prime} s$ and the remaining element of the set, say $x_{k}$. Then,

$x_{i} \equiv \phi_{i}\left(x_{1}, \ldots, x_{n-1}, x_{k}\right) \cdot\left\{\begin{array}{l}i=1, \ldots, n \\ i \neq k\end{array}\right.$

(iii) Substitute Egs. (12) into $\mathrm{g}_{\mathrm{k}}(\mathrm{x})$ so that the function $g_{k}\left(x_{k} ; x_{1}, \ldots, x_{n-2}\right)$ is defined. Then, by Eqs. (10)

$x_{n}=\int \frac{d x_{k}}{g_{k}\left(x_{k} ; x_{1}, \ldots, x_{n-1}\right)} \equiv \psi_{n}\left(x_{k}, x_{1}, \ldots, x_{n-1}\right)$,

where $x_{1}, \ldots, x_{n-1}$ are constants for the gystem defined by Eqs. (10). Then, solving for $\mathrm{x}_{\mathrm{k}}$ in $\mathrm{Eq}$. (13) the function $\mathrm{x}_{\mathrm{k}}=$ $\phi_{\mathrm{k}}\left(\mathrm{x}_{1}, \ldots, \mathrm{x}_{\mathrm{n}}\right)$ is determined. This func tion and Eqs. (12) define the desired point-transformation.

After this method has been applied a new Hamiltonian system $\{\mathrm{X}, \Lambda\}$ with Ham iltonian

$$
K(X, \Lambda, t) \equiv H[x(X), \lambda(X, \Lambda), t]
$$

is defined. In this system $\frac{\partial \mathrm{K}}{\partial \mathrm{X}_{\mathrm{n}}}=-\dot{\Lambda}_{\mathrm{n}}=0$ so $\mathrm{X}_{\mathrm{n}}$ does not appear in $K(X, \Lambda, t)$. Thus, $X_{n}$ will not appear in any of the Hamilton's equations and one need not even integrate the $\dot{X}_{n}$-equation if the time-history of $\mathrm{X}_{\mathrm{n}}$ is not a necessary part of the problem.

The method described above can be used to generate the cylindrical and spherical systems since they possess a conjugate constant of the motion Lagrange multiplier. However, the main reason for presenting the method is that it may prove useful in the generation of nonstandard coordinate systems (e.g., new orbital parameter systems) which possess a conjugate constant of the motion Lagrange multiplier.

IV. Application of Poisson Brackets in the Determination of the Total Canonical Transformation

In the previous section we found that there exist $m$ any canonical transformations which cause one of the new multipliers to be a constant of the motion. If the original system is denoted by $\left\{x_{1}, \ldots, x_{n}\right.$, $\left.\lambda_{1}, \ldots, \lambda_{n}\right\}$ and the new system by $\left\{x_{1}, \ldots, x_{n}\right.$, $\left.\Lambda_{1}, \ldots, \Lambda_{n}\right\}$, then the total Hamiltonian is, for example,

$$
H\left(t, X_{2}, X_{3}, \ldots, X_{n}, \Lambda_{1}, \ldots, \Lambda_{n}\right) \text {, }
$$

where the three constants of the motion are of the form

$$
\begin{aligned}
& A_{1}=\Lambda_{1} \\
& A_{2}=\sum_{i=1}^{n} G_{i 2}\left(X_{1}, \ldots, X_{n}\right) \Lambda_{i}
\end{aligned}
$$

$$
A_{3}=\sum_{i=1}^{n} G_{i 3}\left(x_{1}, \ldots, x_{n}\right) \Lambda_{i} .
$$

Now we wish to perform a canonical transformation in which $\Lambda_{1}$ is invariant and two of the remaining new variables depend only upon $A_{1}, A_{2}$, and $A_{3}$ (at most).

The following theorem gives us a great deal of information concerning such a transformation.

THEOREM IV.1: Let $O(X, \Lambda, t), P(X, \Lambda, t)$ be a canonical transformation such that $P_{1}=\Lambda_{1}$. Then,

(i) $Q_{2}, \ldots, Q_{n}, P_{2}, \ldots, P_{n}$ cannot depend upon $\mathrm{X}_{1}$, and

(ii) $Q_{1}$ depends upon $X_{1}$ linearly, i.e., $Q_{1}=$ $\mathrm{cX}_{1}+\mathrm{f}\left(\mathrm{X}_{2}, \ldots, \mathrm{X}_{\mathrm{n}}, \Lambda_{1}, \ldots, \Lambda_{n}\right)$, where $\mathrm{c}$ is a nonzero constant.

Proof: (i) By Property Il.1. (i), i.e., the Poisson bracket condition, it is necessary that $\left\{P_{1}, Q_{j}\right\}=$ $\left\{P_{1}, P_{j}\right\}=0$ for each $j=2, \ldots, n$. But,

$$
\begin{aligned}
& \left\{P_{1}, Q_{j}\right\}=\left\{\Lambda_{1}, Q_{j}\right\}=\frac{\partial Q_{j}}{\partial X_{1}}=0 \\
& \left\{P_{1}, P_{j}\right\}=\left\{\Lambda_{1}, P_{j}\right\}=\frac{\partial P_{j}}{\partial X_{1}}=0 .
\end{aligned}
$$

Thus, $Q_{2}, \ldots, Q_{n}, P_{2}, \ldots, P_{n}$ cannot depend upon $X_{1}$. (ii) Again by the Poisson bracket theorem it is necessary that

$$
\left\{P_{1}, Q_{1}\right\}=c \text {, }
$$

where $c$ is a nonzero scalar constant. Thus,

$$
\left\{P_{1}, Q_{1}\right\}=\left\{\Lambda_{1}, Q_{1}\right\}=\frac{\partial Q_{1}}{\partial X_{1}}=c \text {, }
$$

which implies

$$
Q_{1}=c X_{1}+f\left(X_{2}, \ldots, X_{n}, \Lambda_{1}, \ldots, \Lambda_{n}\right) \text {. }
$$

With regard to a canonical transformation such that $P_{1}=\Lambda_{1}$ and two of the remaining variables depend only upon $A_{1}, A_{2}$, and $A_{3}$ (at most), this theorem and Theorem III. 1 imply the following:

(1) If $P_{k}, P_{\ell}$, or $P_{k}, Q_{\ell}(k \neq \ell ; k, \ell \in\{2,3, \ldots, n\})$ are the two desired canonical variables, then the nonlinear combinations of $A_{1}\left(\Lambda_{1}\right), A_{2}(X, \Lambda)$, $A_{3}(X, \Lambda)$ which form them cannot depend upon $X_{i}$ Thus, if $A_{2}$ and/or $A_{3}$ depend upon $X_{1}$, then the nonlinear combination must be formed in such a way that $X_{1}$ is elim inated.

(2) If $Q_{1}, P_{\ell}$ or $Q_{1}, Q_{\ell}(\ell=2,3, \ldots$, or $n)$, where $Q_{1}=c X_{1}+f\left(X_{2}, \ldots, X_{n}, \Lambda_{1}, \ldots, \Lambda_{n}\right)$, are the two desired canonical variables, then the nonlinear combination of the $A_{i}{ }^{\prime} s$ which forms $P_{\ell}$ or $Q_{\ell}$ $\left(\ell \neq 1\right.$ ) cannot depend upon $X_{1}$, and $Q_{1}$ must be formed from the $A_{i}{ }^{\prime} s$ in such a way that $X_{1}$ appears linearly.

Properties (1) and (2) mentioned above restrict considerably the possible choices for three of the new canonical variables (i.e., $P_{1}$ and two other variables). In the next section we shall see that the 
obvious choices for nonlinear combinations of the $A_{i}^{\prime}$ s which satisfy (1) and (2) lead to the desired canonical transformation.

\section{Canonical Systems with Three Constant of the Motion Components}

In this section a new canonical system, which contains three constant of the motion components, will be determ ined for the optimal trajectory problem. From Theorem III. 1 we know that such a system must contain at least two nonlinear combinations of $A_{1}, A_{2}$, and $A_{3}$ (where $A_{1} \bar{i}+A_{2} \bar{j}+A_{3} \bar{k}$ is the known vector constant of the motion).

Later in this section it will be shown that the $A_{i}$ 's can be expressed in spherical coordinates as

$$
\begin{aligned}
& A_{1}=A \cos \phi-B \sin \phi \\
& A_{2}=A \sin \phi+B \cos \phi \\
& A_{3}=\lambda_{6},
\end{aligned}
$$

where $\phi$ is the coordinate canonically conjugate to the constant multiplier $\lambda_{6}$ and neither $A$ nor $B$ depends upon $\phi$. (With respect to Section IV, $\lambda_{6}$ corresponds to $\Lambda_{1}$ and $\phi$ corresponds to $X_{1}$.) By Theorem IV. 1 at least one of the nonlinear combinations of the $A_{i}{ }^{\prime} s$, which is to be a new canonical variable, cannot depend upon $\phi$. Inspection of Eqs. (17) suggests two basic functional forms: $f\left(A_{1}^{2}+A_{2}^{2}\right)$ or $f\left(A_{1}^{2}+A_{2}^{2}+A_{3}^{2}\right)$. The simplest of these forms are $A_{1}^{2}+A_{2}^{2}, \sqrt{A_{1}^{2}+A_{2}^{2}}, A_{1}^{2}+A_{2}^{2}+$ $A_{3}^{2}$, and $\sqrt{A_{1}^{2}+A_{1}^{2}+A_{3}^{2}}$. With the canonical transformation technique of this paper (to be discussed later), $\sqrt{A_{1}^{2}+A_{2}^{2}}$ does not allow the desired trans formation whereas $\sqrt{A_{1}^{2}+A_{2}^{2}+A_{3}^{2}}$ does. Thus, $\sqrt{A_{1}^{2}+A_{2}^{2}+A_{3}^{2}}$ will be one of our new momenta variables. (Note that by performing a simple canonical transformation $\sqrt{A_{1}^{2}+A_{2}^{2}+A_{3}^{2}}$ could alternatively be a new generalized coordinate.)

Finally, consider the possibilities for the third new constant of the motion canonical variable. Since there does not exist another functionally independent, with respect to $f\left(A_{1}^{2}+A_{2}^{2}+A_{3}^{2}\right)$, nonlinear combination of the $\mathrm{A}^{\prime} \mathrm{s}$ s which does not contain $\phi$, then the only possibility for the third variable is a linear function $\phi$ and it must be canonically conjugate to $\lambda_{6}=A_{3}$ (by Theorem IV.1). At first glance it does not appear that a nonlinear combination of the $\mathrm{Ai}^{\dagger} \mathrm{s}$ can form a linear function of $\phi$. However, $\tan ^{-1}\left(A_{1} /-A_{2}\right)$ is such a function, and is indeed the desired third canonical variable. Since the Hamilton-Jacobi theory is used to deter$m$ ine the transformation, the new generalized coordinates result from simple differentiations. Thus, it appears that $\tan ^{-1}\left(A_{1} /-A_{2}\right)$ would simply "fall out" and, would not be useful in generating the transformation. This is not the case since the requirement that the generalized coordinate conjugate to $\lambda_{6}=A_{1}$ is also a constant of the motion is the means by which one chooses the proper functional form $f\left(A_{1}^{2}+A_{2}^{2}+A_{3}^{2}\right)$. (That is, it was found that $f\left(A_{1}^{2}+A_{2}^{2}\right)$ does not produce a constant of the motion conjugate to $\lambda_{6}$, whereas $f\left(A_{1}^{2}+A_{2}^{2}+A_{3}^{2}\right)$ does.)
Before the desired canonical transformation is effected, let us consider the possible ways in which we can generate the transformation. First of all note that since the constants of the motion depend upon all of the original variables, i.e., $A_{1}(x, \lambda)$, $A_{2}(x, \lambda), A_{3}(x, \lambda)$, then the possibility of performing simple transformations and using independence arguments $^{(2)}$ is not applicable, i.e., the system $\{x, \lambda\}$ cannot be transformed into an intermediate system $\{\mathrm{X}, \Lambda\}$ such that $\delta \mathrm{S}=\sum_{\mathrm{i}=1}^{\mathrm{n}}\left(\Lambda_{\mathrm{i}} \delta \mathrm{X}_{\mathrm{i}}-\mathrm{P}_{\mathrm{i}} \delta \mathrm{Q}_{\mathrm{i}}(\mathrm{X})\right)$ can be used to define the transformation by independence arguments.

Another approach which is possible but will not be pursued here is the following. We know by Definition II. 1 that a canonical transformation is independent of the Hamiltonian function, and by Eq. (3) that

$$
\frac{\partial S}{\partial t}+H\left(x, \frac{\partial S}{\partial x}, t\right)=0
$$

implies a canonical transformation which results in the equilibrium solution for the system defined by $\mathrm{H}\left(\mathrm{x}, \frac{\partial \mathrm{S}}{\partial t}, \mathrm{t}\right)$. Thus, the desired transformation can be obtained by solving the partial differential equation (18) for any Hamiltonian such that $\sqrt{\bar{A}_{1}^{2}+A_{2}^{2}+A_{3}^{2}}$ and $A_{3}$ are constants of the motion for the corresponding Hamiltonian system. Since $A_{1}^{2}+A_{2}^{2}$ does not depend upon $\phi$, then such a Hamiltonian is

$$
H=A_{1}^{2}(\widetilde{x}, \lambda)+A_{2}^{2}(\widetilde{x}, \lambda)+\lambda_{6}^{2},
$$

where $\tilde{x}$ does not depend upon $\phi$. Since this $H$ does not depend upon time it $m$ ust be a constant of the motion ( say $\alpha_{1}^{2}$ ) and since $\phi$ does not appear explicitly, then $\lambda_{6}$ must be a constant of the motion (say $\left.\alpha_{2}\right)$. To complete the canonical transformation, one must determine a complete solution $S\left(t, x_{1}, \ldots, x_{n}\right.$, $\left.\alpha_{1}, \ldots, \alpha_{n}\right)$ of the Ham ilton-Jacobi equation

$$
-\alpha_{1}^{2}+A_{1}^{2}\left(\tilde{x}, \frac{\partial S}{\partial \widetilde{x}}\right)+A_{2}^{2}\left(\widetilde{x}, \frac{\partial S}{\partial \widetilde{x}}\right)+\alpha_{2}^{2}=0 .
$$

The new momenta variables are $\alpha_{1}, \ldots, \alpha_{n}$ and the new generalized coordinates are $\beta_{i}=\frac{\partial S}{\partial x_{i}}(i=1, \ldots, n)$.

The approach mentioned above is undesirable because the Hamiltonian of Eq. (19) is not physically motivated and the Hamilton-Jacobi equation of Eq. (20) is not easy to solve. Therefore, since we must solve a nontrivial partial differential equation, we should try to obtain as much physical knowledge about the problem as possible. Since $\sqrt{A_{1}^{2}+A_{2}^{2}+A_{3}^{2-}}$ and $A_{3}$ are also constants of the motion for the optimal trajectory problem with zero-thrust (i.e., the coast-arc problem), then a logical choice for the Hamiltonian is the zero-thrust Hamiltonian. In this case the new canonical system $\{\alpha, \beta\}$ will have two desirable properties: (1) two of the new momenta variables and one of the new generalized coordinates will be constants of the motion for the total optimal trajectory problem; and (2) all of the variables $\alpha_{1}, \ldots, \alpha_{n}, \beta_{1}, \ldots, \beta_{n}$ are constants of the motion for the coast-arc problem. Thus, for low-thrust $\mathrm{m}$ issions the set $\{\alpha, \beta\}$ should be a slowly varying set of variables. 
The equations of motion for a continuously thrusting rocket in an inverse square gravitational force field are

$$
\begin{aligned}
\dot{\mathrm{u}}= & \frac{\mathrm{v}^{2}}{\mathrm{r}^{3}}+\frac{\mathrm{w}^{2}}{\mathrm{r}^{3} \cos ^{2} \theta}-\frac{\mathrm{k}}{\mathrm{r}^{2}} . \\
& +\frac{F}{\mathrm{~m}}\left[\cos \theta \cos \tau \cos \chi^{*}+\sin \tau \sin \theta\right] \\
\dot{\mathrm{v}}= & -\frac{\mathrm{w}^{2}}{\mathrm{r}^{2}} \sec ^{2} \theta \tan \theta \\
& +\mathrm{r} \frac{\mathrm{F}}{\mathrm{m}}\left[\sin \tau \cos \theta-\cos \tau \sin \theta \cos \chi^{*}\right] \\
\dot{\mathrm{w}}= & \mathrm{r} \frac{\mathrm{F}}{\mathrm{m}} \cos \tau \cos \theta \sin \chi^{*} \\
\dot{\mathrm{r}}= & \mathrm{u} \\
\dot{\theta}= & \frac{\mathrm{v}}{\mathrm{r}^{2}} \\
\dot{\phi}= & \frac{\mathrm{w}}{\mathrm{r}^{2} \cos ^{2} \theta} \\
\dot{\mathrm{m}}= & -\sigma
\end{aligned}
$$

where the variables $r, \theta, \phi, \tau$, and $X$ are defined in Figure $I$ and $X * \equiv X-\phi$. The thrust magnitude, $F$, and mass flow rate parameter, $\sigma$, are assumed constant. Upon application of the maximum principle (assuming that a scalar quantity $g\left(t_{f}, x_{f}\right)$ is to be minimized), the control variables are determined as functions of state variables and Lagrange multipliers, and the following generalized Hamiltonian describes the problem:

$$
\begin{aligned}
H= & \lambda_{1}\left(\frac{v^{2}}{r^{3}}+\frac{w^{2}}{r^{3}} \sec ^{2} \theta-\frac{k}{r^{2}}\right) \\
& -\lambda_{2} \frac{w^{2}}{r^{2}} \sec ^{2} \theta \tan \theta \\
& +\lambda_{4} u+\lambda_{5} \frac{v}{r^{2}}+\lambda_{6} \frac{w}{r^{2}} \sec ^{2} \theta-\lambda_{7} \sigma \\
& +\frac{F}{m} \sqrt{\lambda_{1}^{2}+r^{2} \lambda_{2}^{2}+r^{2} \lambda_{3}^{2} \cos ^{2} \theta}
\end{aligned}
$$

In this formulation the three components of the vector integral become

$$
\begin{aligned}
& A_{1} \equiv A \cos \phi-B \sin \phi \\
& A_{2} \equiv A \sin \phi+B \cos \phi \\
& A_{3}=\lambda_{6},
\end{aligned}
$$

where

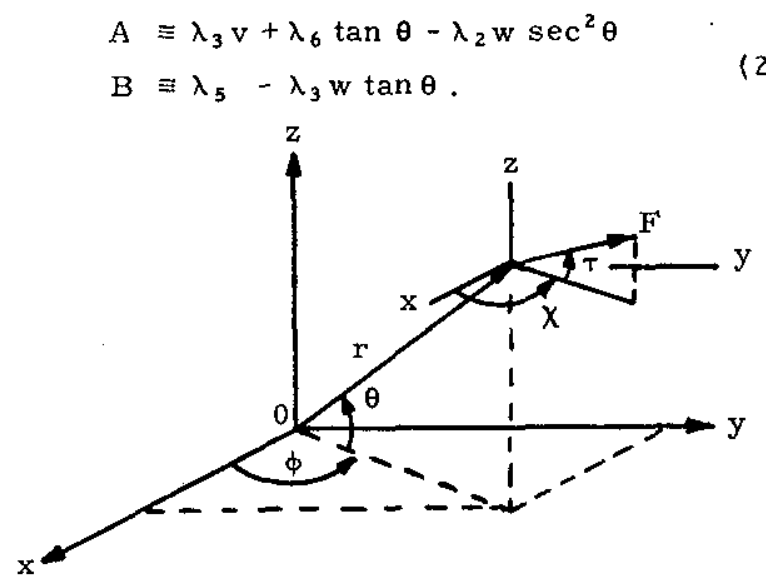

Figure 1. Geometry and Control Angle Definition
In References 2 and 5, the application of simple canonical transformations to the solution of Hamilton-Jacobi equations is discussed. Based on those results the following simple canonical transformation is defined for the problem considered here:

$Q_{1}=\lambda_{1}, Q_{2}=\lambda_{2}, Q_{3}=w, Q_{4}=r, Q_{5}=\theta, Q_{6}=\phi, Q_{7}=m$

$P_{1}=-u, P_{2}=-v, P_{3}=\lambda_{3}, P_{4}=\lambda_{4}, P_{5}=\lambda_{5}, P_{6}=\lambda_{6}, P_{7}=\lambda_{7}$,

where the $Q_{i}{ }^{\prime} s$ and $P_{i}{ }^{\prime} s$ represent new generalized coordinates and momenta, respectively. The coast-arc Hamilton-Jacobi equation (i.e., the Hamilton-Jacobi equation for the Hamiltonian of Eq. (22) with $F=0$ ) is then:

$$
\begin{aligned}
& \frac{\partial S}{\partial t}+Q_{1}\left[\frac{S_{2}^{2}+Q_{3}^{2} \sec ^{2} Q_{5}}{Q_{4}^{3}}-\frac{k}{Q_{4}}\right] \\
& -\frac{Q_{2} Q_{3}^{2}}{Q_{4}^{2}} \sec ^{2} Q_{5} \tan Q_{5} \\
& -S_{4} S_{1}-\frac{S_{5} S_{2}}{Q_{4}^{2}}+\frac{S_{6} Q_{3} \sec ^{2} Q_{5}}{Q_{4}^{2}}-\sigma S_{7}=0,
\end{aligned}
$$

where $S_{i} \equiv \frac{\partial S}{\partial Q_{i}}$. A complete solution of this equation will now be effected, and two of the new momenta variables, $\alpha_{3}$ and $\alpha_{7}$, will be strictly functions of $A_{1}, A_{2}$, and $A_{3}$.

Since neither $t, Q_{7}$, nor $Q_{6}$ appear explicitly in Eq. (26), then $\partial S / \partial t, S_{7}$, and $S_{6}$ must be constants of the motion, say $\alpha_{1}, \alpha_{2} / \sigma$, and $\alpha_{3}$. From knowledge of the two-body problem (which describes the state on the coast-arc) two more constant relationships are known:

$$
\begin{aligned}
\alpha_{4}^{2} & =\mathrm{v}^{2}+\mathrm{w}^{2} \sec ^{2} \theta \\
& =\mathrm{S}_{2}^{2}+\mathrm{Q}_{3}^{2} \sec ^{2} \mathrm{Q}_{5} \quad \text { (angular momentum) } \\
-\alpha_{5} & =\mathrm{u}^{2}+\frac{\alpha_{4}^{2}}{\mathrm{r}^{2}}-\frac{2 \mathrm{k}}{\mathrm{r}} \\
& =\mathrm{S}_{1}^{2}+\frac{\alpha_{4}^{2}}{Q_{4}^{2}}-\frac{2 \mathrm{k}}{Q_{4}} \cdot \text { (energy) }
\end{aligned}
$$

Upon substitution of $\partial \mathrm{S} / \partial \mathrm{t}=\alpha_{1}, \mathrm{~S}_{7}=\alpha_{2} / \sigma, \mathrm{S}_{6}=\alpha_{3}$, $S_{I}(\alpha, Q)$, and $S_{2}(\alpha, Q)$ into Eq. (26) another separation of variables can be performed so that a sixth constant $\alpha_{6}$ may be defined such that both of the following equations hold:

$$
\begin{aligned}
\alpha_{6} \alpha_{4}= & \left(\alpha_{1}-\alpha_{2}\right) Q_{4}^{2}+Q_{1}\left(\frac{\alpha_{4}^{2}}{Q_{4}}-k\right) \\
& -Q_{4} S_{4} \sqrt{2 k Q_{4}-\alpha_{5} Q_{4}^{2}-\alpha_{4}^{2}} \\
\alpha_{6} \alpha_{4}= & Q_{2} Q_{3}^{2} \sec ^{2} Q_{5} \tan Q_{5}-\alpha_{3} Q_{3} \sec ^{2} Q_{5} \\
& -S_{5} \sqrt{\alpha_{4}^{2}-Q_{3}^{2} \sec ^{2} Q_{5}} .
\end{aligned}
$$

A more detailed discussion of the solution technique outlined above may be found in Reference 6. Also, it should be noted that \pm signs have been omitted in the determination of $S_{1}$ and $S_{2}$ from Eqs. (27). The consequences of this will be discussed later.

For a complete solution of Eq. (26), seven constants are required and so far we have obtained 
only six. However, we have not made use of the $\sqrt{A_{1}^{2}+A_{2}^{2}+A_{3}^{2}}$ constant. With the constants that we already know we can use the solution technique of References 5 and 6 to form an incomplete solution of Eq. (26) of the form

$$
S *\left(t, Q_{1}, \ldots, Q_{7}, \alpha_{1}, \ldots, \alpha_{6}\right) \text {. }
$$

To form the complete solution we note that Eq. (26) does not contain an $S_{3} \equiv \partial S / \partial Q_{3}$-term (this is a result of the base Hamiltonian not containing $\lambda_{3}$ ). Thus, the addition of any function of $Q_{3}$ to $S *$ will not affect the Ham ilton-Jacobi equation, i.e., $S^{*}$ $+f\left(Q_{3}, \alpha\right)$ is also a solution of $\mathrm{Eq} .(26)$. Instead of picking an arbitrary function of $Q_{3}$, we shall choose the one which results from defining $\alpha_{7}^{2}=\mathrm{A}_{1}^{2}$ $+\mathrm{A}_{2}^{2}+\mathrm{A}_{3}^{2}$.

Upon evaluation of $A_{1}^{2}, A_{2}^{2}$, and $A_{3}^{2}$ in terms of $S_{3}, Q_{i}^{\prime} s$ and $\alpha_{i}^{\prime} s$ a quadratic in $S_{3}$ is formed, and then

$$
\begin{aligned}
S_{3}= & \frac{Q_{3} g_{2} \tan Q_{5}-g_{1} S_{2}\left(Q_{3}, Q_{5}, \alpha_{4}\right)}{\left(\alpha_{4}^{2}-Q_{3}^{2}\right)} \\
& \pm \frac{\sqrt{\left(\alpha_{7}^{2}-\alpha_{3}^{2}\right)\left(\alpha_{4}^{2}-Q_{3}^{2}\right)-\left(\alpha_{3} Q_{3}+\alpha_{4} \alpha_{6}\right)^{2}}}{\left(\alpha_{4}^{2}-Q_{3}^{2}\right)}
\end{aligned}
$$

where

$$
\begin{aligned}
& g_{1} \equiv \alpha_{3} \tan Q_{5}-Q_{2} Q_{3} \sec ^{2} Q_{5} \\
& g_{2} \equiv S_{5}=\left[Q_{2} Q_{3}^{2} \sec ^{2} Q_{5} \tan Q_{5}\right. \\
&\left.-\alpha_{3} Q_{3} \sec ^{2} Q_{5}-\alpha_{4} \alpha_{6}\right] / S_{2}\left(Q_{3}, Q_{5}, \alpha_{4}\right)
\end{aligned}
$$

and use has been made of the relationship

$$
g_{1} Q_{3} \tan Q_{5}+g_{2} S_{2}\left(Q_{3}, Q_{5}, \alpha_{4}\right)=-\left(\alpha_{3} Q_{3}+\alpha_{4} \alpha_{6}\right) \text {. }
$$

Note that Eq. (31) has the following functional form

$$
S_{3}=f_{1}\left(\alpha ; Q_{2}, Q_{3}, Q_{5}\right) \pm f_{2}\left(\alpha, Q_{3}\right) \text {. }
$$

If one differentiates $S *$ of $\mathrm{Eq} .(30)$ with respect to $Q_{3}$, then $f_{1}\left(\alpha ; Q_{2}, Q_{3}, Q_{5}\right)$ will be the result. Thus, the function of $Q_{3}$ which we wish to adjoin to $S *$ is just the indefinite integral of $f_{2}\left(\alpha, Q_{3}\right)$. Evaluating this integral and writing out the $S^{*}$-function, we obtain the following complete solution to the Hamilton-Jacobi equation (26):

$$
\begin{aligned}
& S=\alpha_{1} t+\alpha_{2} Q_{7}+\alpha_{3}\left[Q_{6}-\sin ^{-1} \frac{Q_{3} \tan Q_{3}}{\sqrt{\alpha_{4}^{2}-Q_{3}^{2}}}\right] \\
& -Q_{1} \sqrt{\frac{2 k}{Q_{4}}-\frac{\alpha_{4}^{2}}{Q_{4}^{2}}-\alpha_{5}}-Q_{2} \sqrt{\alpha_{4}^{2}-Q_{3}^{2} \sec ^{2} Q_{5}} \\
& +\alpha_{6}\left[\cos ^{-1} \frac{\alpha_{4}^{2}-\mathrm{kQ}_{4}}{Q_{1} \sqrt{\mathrm{k}^{2}-\alpha_{4}^{2} \alpha_{5}}}-\sin ^{-1} \frac{\alpha_{4} \sin Q_{5}}{\sqrt{\alpha_{4}^{2}-Q_{3}^{2}}}\right] \\
& +\left(\alpha_{1}-\alpha_{2}\right)\left[\frac{Q_{1}}{\alpha_{5}} \sqrt{\frac{2 \mathrm{k}}{Q_{4}}-\frac{\alpha_{4}^{2}}{Q_{4}^{2}}-\alpha_{5}}-\frac{\mathrm{k}}{\alpha_{5}^{\frac{3}{2}}} \cos ^{-1} \frac{\mathrm{k}-\alpha_{5} \mathrm{Q}_{4}}{\sqrt{\mathrm{k}^{2}-\alpha_{4}^{2} \alpha_{5}}}\right] \\
& +\left\{\alpha_{7} \sin ^{-1} \frac{\alpha_{7}^{2} Q_{3}+\alpha_{3} \alpha_{4} \alpha_{6}}{\alpha_{4} \sqrt{\alpha_{7}^{2}-\alpha_{3}^{2}} \sqrt{\alpha_{7}^{2}-\alpha_{6}^{2}}}\right. \\
& +\alpha_{3}: \cos ^{-1} \frac{\alpha_{4} \alpha_{6}+\alpha_{3} Q_{3}}{\sqrt{\alpha_{4}^{2}-Q_{3}^{2}} \sqrt{\alpha_{7}^{2}-\alpha_{3}^{2}}} \\
& \left.+\alpha_{6} \cos ^{-1} \frac{\alpha_{3} \alpha_{1}+\alpha_{6} Q_{3}}{\sqrt{\alpha_{1}^{2}-Q_{3}^{2}} \sqrt{\alpha_{7}^{2}-\alpha_{6}^{2}}}\right\}
\end{aligned}
$$

where $\{\ldots\}$ is the $f_{2}\left(\alpha, Q_{3}\right)$ contribution. The seven remaining canonical variables may be obtained by applying Jacobi's Theorem, i.e., $\beta_{i}=\partial S / \partial \alpha_{i}$ $(i=1, \ldots, 7)$. Since these new variables $c$ an be obtained by differentiation alone, we shall only develop $\beta_{3}=\partial S / \partial \alpha_{3}$ to show that it is equal to $\tan ^{-I}\left(A_{1} /-A_{2}\right)$. gives

Differentiation of Eq. (35) with respect to $\alpha_{3}$

$$
\begin{aligned}
\beta_{3}= & \frac{\partial S}{\partial \alpha_{3}}=Q_{6}-\sin ^{-1} \frac{Q_{3} \tan Q_{5}}{\sqrt{\alpha_{4}^{2}-Q_{3}^{2}}} \\
& +\cos ^{-1} \frac{\alpha_{4} \alpha_{6}+\alpha_{3} Q_{3}}{\sqrt{\alpha_{4}^{2}-Q_{3}^{2}} \sqrt{\alpha_{7}^{2}-\alpha_{3}^{2}}} .
\end{aligned}
$$

If one combines the last two terms of Eq. (36) to form a single $\sin ^{-1}$-function and also combines them to form a single $\cos ^{-1}$-function, and then makes use of the following equalities:

$\alpha_{4} \alpha_{6}+\alpha_{3} w=-w\left[\lambda_{3} v-\lambda_{2} w \sec ^{2} \theta+\lambda_{6} \tan \theta\right] \tan \theta$

$\mathrm{v}\left(\lambda_{5}-\lambda_{3} \mathrm{w} \tan \theta\right)=\mathrm{Aw} \tan \theta+\mathrm{BV}$

$\alpha_{7}^{2}-\alpha_{3}^{2}=\mathrm{A}^{2}+\mathrm{B}^{2}$

$\alpha_{4}^{2}-\mathrm{w}^{2}=\mathrm{v}^{2}+\mathrm{w}^{2} \tan ^{2} \theta$

$\left(\alpha_{4}^{2}-Q_{3}^{2}\right)\left(\alpha_{7}^{2}-\alpha_{3}^{2}\right)-\left(\alpha_{4} \alpha_{6}+\alpha_{3} w\right)^{2}=(v A-w B \tan \theta)^{2}$

then the following expressions are valid:

$\sin \left(\beta_{3}-\phi\right)=\frac{A}{\sqrt{A^{2}+B^{2}}}, \cos \left(\beta_{3}-\phi\right)=\frac{-B}{\sqrt{A^{2}+B^{2}}}$.

From these equations and Eqs. (23) it follows that

$$
\tan \beta_{3}=\frac{A_{1}}{-A_{2}} \text {. }
$$

Therefore, the system $\{\alpha, \beta=\partial S / a \beta\}$ represents a set of canonical variables which are canonic constants for the coast-arc problem and $\alpha_{3}, \alpha_{7}$, and $\beta_{3}$ are strictly functions of $A_{1}, A_{2}$, and $A_{3}$ so they are constants of the motion for the total problem.

\section{Concluding Remarks}

The present study has sought to extend the applicability of the known vector integral for the optimal trajectory problem. It was shown that a classic theorem due to Whittaker can be used to define a large class of state variables such that a conjugate Lagrange multiplier is a component of the vector integral. Also, a canonical transformation was used to define a new canonical system in which three of the variables are constants of the motion. Although the resultant system is cumbersome, it demonstrates the existence of a canonical system with constant of the motion components for the coast-arc problem such that three of the components are constants for the total problem. Since the primary goal here was to generate such a transformation, the \pm signs which result from solving quadratic equations throughout the analysis were dropped (thus the resultant solution is only valid for the positive case). However, now that it is known that such a transformation exists, the \pm sign difficulty should be removable in a manner similar 
to the way that the Hamilton-Jacobi solution of Reference 2 removed the \pm difficulty in the solutions of References 5 and 6.

\section{$\underline{\text { References }}$}

1. Pines, S., "Constants of the Motion for Optimum Thrust Trajectories in a Central Force Field," AlAA Journal, November 1964, pp. 2010-2014.

2. Powers, W. F. and Tapley, B.D. "Canonical Transformation Applications to Optimal Trajectory Analysis," AIAA Journal, March 1969, pp. 394-399.

3. Whittaker, E.T., Analytical Dynamics of Particles and Rigid Bodies (Fourth Edition), Cambridge University Press, London, 1964, Section 150 .
4. Caratheodory, C, , Calculus of Variations and Partial Differential Equations of the First Order: Part I (translated by R. B. Dean and J.J. Brand$\overline{\text { statter) }}$, Holden-Day, Inc., San Francisco, 1965, pp. 26-30.

5. Miner, W.E., Tapley, B.D., and Powers, W. F., "The Hamilton-Jacobi Method Applied to the Low-Thrust Problem, " Proceedings of the 18th Congress of the International Astronautical Federation, Belgrade, Yugoslavia, September 1967, Pergamon Press, pp. 293-305.

6. Powers, W. F. and Tapley, B. D., "Canonical Transformation Theory and the Optimal Trajectory Problem," Univ. of Texas Engineering Mechanics Research Lab Report TR-1022, August 1967, pp. 70-80. 